research. Working Group 3 aims to identify and prioritize tools that can improve efficiency in producing and updating systematic reviews. Working Group 4 will explore methods to detect redundant research as well as measurable outcomes of implementing evidence-based research approach that are relevant to researchers and key stakeholders.

Conclusions The EVBRES imitative, a COST Action Network, was initiated by the EBRNetwork, and successfully launched and is funded to carry out activities until October 2022. The EBRNetwork and its work will play a crucial role in increasing systematic use of existing evidence when planning new research and interpreting the results. These initial steps will lay the firm foundations for future endeavour to promote evidence-based research.

\section{HIERARCHICAL ARRANGEMENT OF SCHOLARLY AND NOVEL INFORMATION (HASANI): A DIGITAL PLATFORM TO SYNTHESIZE RESEARCH EVIDENCE IN REAL TIME}

${ }^{1}$ Izhar Hasan, 'Uzair Hasan, ${ }^{2}$ Babar Rao, 'Salman Habib Abbasi. 'MDACCESS, Princeton, USA; ${ }^{2}$ Robert wood Jhonson Medical school, Somerset, USA

10.1136/bmjebm-2019-EBMLive. 17

Objectives Our main objective is to create a real time evidence generating platform for the most uptodate evidence-based practice with following specific goals.

1. Implement a digital curation framework to automate knowledge extraction and summerization of published and unpublished publications

2. Implement a critical appraisal process at the time of publication submission to journals.

3. Implement an integrated visual abstract application for authors to submit critically appraised elements of the study

4. Implement an automated data analytical interface to generate automated systematic reviews and meta-analysis

5. Implement a platform API for publishers to automatically summarize their published papers for automated systematic reviews and met analysis.

Method We designed and implemented a platform for automating the research evidence synthesis. The application is implemented as SaaS-based model in angular JS framework with Application program interface (API) to integrate with online journals. This creates an opportunity to synthesize research evidence in real time for a given research topic, population or territory. Each publisher or organization can customize templates for various study types to create automated systematic review, meta-analysis, and qualitative review studies Results A beta testing of our platform (HASANI) has been conducted to display the automated article summary creation from critical appraisal elements. Preliminary beta study results confirmed that our framework was efficient in identifying, curating and synthesizing the literature article summary of a given article to pool against similar studies. This strategy not only saves time and money in synthesizing new research evidence, but also provides a platform for insuring a quality research publication as it eliminates a human bias and errors. A real time automated pooling of similar research studies expedites a creation of automated systematic reviews generation which addresses the research publication overload in coming years

Conclusions We have designed and implemented a web-based automated literature reading and curating framework for research evidence synthesis in real time. Our initial findings provide supportive evidence of automating the literature curating and extraction strategy. In addition, it provides an excellent future digital curation strategy for journal publishers to streamline the synthesis of research evidence by requesting authors to submit the pre-appraised critical elements of the relevant study types. This strategy provides a reassurance to academic audience that published articles have been formally appraised to be included in building a research evidence towards a particular topic or subject. More importantly, a real-time knowledge synthesis from this strategy will provide a more robust and uptodate practice guidelines for clinicians to focus on interpretation of research findings for applicability in their patient population, rather waiting for new systematic review and meta-analysis creation.

\section{RCTS: WHAT ELSE? TEACHING RESEARCH METHODS WITH A CAFFEINE BOOST}

Luigia Scudeller, Cristina Capittini, Annalisa De Silvestri, Catherine Klersy, Valeria Musella, Carmine Tinelli. Scientific Direction, Fondazione IRCCS Policlinic San Matteo, PAVIA (PV), Italy

\subsection{6/bmjebm-2019-EBMLive.18}

Objectives Randomized Controlled Trials are complex undertakings, involving many different abilities, skills, knowledges and notions, some of which can only be acquired by direct experience. Objective of this teaching experiment was to assess short-term learning efficacy of a simulated RCT on knowledge of methodological issues involved in clinical research.

Method The final exam of our 400-hours course on Methods in Biomedical Research, was a 2-days simulation of a randomized placebo-controlled trial with 30 'patients' in 5 'clinical centres' (Africa, America, Asia, Europe, Oceania), in three editions $(2015-2017$, final sample size $=90)$. The study question was on efficacy of caffeine on cardiovascular endpoints, and safety. Participants were randomized to standard vs decaffeinated Italian espresso in a blinded fashion, and were guided to actively perform:

Day 1, morning: study design,CRF design, trial registration

Day 1, afternoon: implementation (including obtaining consent, randomization, etc)

Day 2, morning: statistical analysis

Day 2, afternoon: study report (CONSORT)

Knowledge was tested pre- and post-intervention by means of a standardized questionnaire, based on the CONSORT checklist, scoring 0-30.

Participants were also blind to coffee manufacturer, and were allowed the following concomitant medications: sugar, milk, biscuits, or chocolates.

The trial was not funded by a coffee maker.

Results Trial participants improved their median knowledge of RTCs methods from 15 points (IQR 10-24) to 28 points 